Article

\title{
Hybrids of Gold Nanoparticles with Core-Shell Hyperbranched Polymers: Synthesis, Characterization, and Their High Catalytic Activity for Reduction of 4-Nitrophenol
}

\author{
Yi Liu, Li Xu, Xunyong Liu * and Mengnan Cao
}

Received: 6 November 2015; Accepted: 18 December 2015; Published: 25 December 2015

Academic Editor: Keith Hohn

School of Chemistry and Materials Science, Ludong University, 264025 Yantai, Shandong, China; liuyi200541@126.com (Y.L.); ldxuli@126.com (L.X.); chenstudent13@126.com (M.C.)

* Correspondence: xunyongliu@126.com; Tel./Fax: +86-535-667-2176

\begin{abstract}
Hyperbranched core-shell structure can be constructed by the modification of hyperbranched polyethylenimine (HPEI) with different amide shells. Functionalized HPEI with acetic amide (HPEI-ACAm), propionic amide (HPEI-PRAm), butyric amide (HPEI-BUAm) and isobutyric amide (HPEI-IBAm) shells have been successfully prepared and used as protectors for gold nanoparticles (AuNPs). Novel AuNP composites were obtained through the non-covalent interaction between HPEI-XXAm and gold nanoparticles (XXAm represents ACAm, PRAm, BUAm or IBAm). The resulted AuNP composites can catalyze the reduction reaction of 4-nitrophenol by $\mathrm{NaBH}_{4}$. Interestingly, the catalytic activity of the AuNPs mainly depends on the structure of protectors and the degree of carbon chain arrangement denseness, which should affect the diffusivity of the reactants. In addition, the order of reaction rate is HPEI10K-IBAm $0.80>$ HPEI10K-ACAm $0.80>$ HPEI10K-PRAm $0.82>$ HPEI10K-BUAm 0.83 . It was found that the increase of the concentrations of the capping HPEI-XXAm polymers can enhance both the reaction rate and the turnover frequency (TOF) values. Furthermore, the reaction rate was accelerated with increasing the reaction temperature for AuNPs-HPEI10K-ACAm 0.80 and AuNPs-HPEI10K-PRAm 0.82 systems. Interestingly, the reaction rate was accelerated with elevating reaction temperature at the beginning but reached a plateau or decreased sharply for AuNPs-HPEI10K-IBAm 0.80 and AuNPs-HPEI10K-BUAm 0.82 systems, owing to the thermoresponsivity of the corresponding AuNP composites. As a consequence, the catalytic activity could be controlled by adjusting the different shells of the hyperbranched polyethylenimine.
\end{abstract}

Keywords: core-shell; catalysis; gold nanoparticle; hyperbranched polymer

\section{Introduction}

Recently, the synthesis and diverse applications of metal nanoparticles with different sizes and shapes have drawn considerable attention [1-4]. Nanoparticles are usually prepared by the reduction of metal ions in the presence of suitable stabilizer like hyperbranced polymers [5], dendrimers [6-8], microgels [9] and surfactants [10] which will prevent the nanoparticles from aggregation. These metal nanoparticles with an organic functional shell [11-14] have attracted much interest due to their functional applications as sensor [15-18], catalyst $[19,20]$ and biological material. In particular, the catalysis is of core interest to chemists. Beyond the enhancement of solubility and the prevention of aggregation, the organic shell can also induce selectivity in catalytic reactions and compatibility in physiological systems [21]. 
Different kinds of capping agents were developed to stabilize the metal nanoparticles [22,23]. Utilization of multifunctional ligands is an effective approach to enhance the stability of stabilizer-protected metal nanoparticles $[24,25]$. The concept heavily relies on the understanding that, for a noble metal nanoparticle protected with a large number of binding groups, the chance of having all binding groups desorbed from the surface of metal nanoparticles simultaneously is definitely much lower than that with only one binding group. Therefore, the stabilization of metal nanoparticles is essential for improving their catalytic activity, especially the ones stabilized by the dendritic polymers (i.e., the dendrimers and hyperbranched polymers). Compared to the perfectly branched dendrimers (degree of branching, DB 100\%), hyperbranched polymers possess a randomly branched topology (degree of branching, DB ca. 60\%). In addition, unlike the tedious multistep syntheses of dendrimers, hyperbranched polymers can be obtained by single-step protocols, indicating attractive potential for applications. Despite the fact that they are not monodisperse like dendrimers, certain hyperbranched polymers can be prepared conveniently with narrow molecular weight distributions. Furthermore, hyperbranched polyethylenimine and polyglycerol are commercially available with narrow molecular weight distributions [5,12]. For the investigations of the solution structures of nanocomposites of highly branched polymers with metal particles, modified polyethylenimines are more suitable than polyglycerols, since the amine functions of the branched scaffold have stronger coordination with metal nanoparticles compared to the polyether-polyol scaffold of the polyglycerols.

Hyperbranched polymers capped metal nanoparticles exhibit high catalytic activity $[5,12,13]$. Inspired by these studies, we became interested in illustrating how a dendritic structure could affect the catalytic behavior of the metal nanoparticles. Herein, we developed the preparation of modified-hyperbranched polyethylenimine protected nanoparticles using a new set of ligands and compared their corresponding catalytic activity. The catalytic characteristics of the composites are tested on the reduction reaction of 4-nitrophenol with $\mathrm{NaBH}_{4}$, which is a well-known model reaction and has been extensively used to evaluate the catalytic rate of metal nanoparticles composites. We attempted to demonstrate the relationship between the stabilizer structure and catalytic activity. Importantly, the catalytic activity could be controlled by adjusting the shell size of the hyperbranched polyethylenimine. To the best of our knowledge, this is the first report about the effect of the structure of hyperbranched polymers on the catalytic behavior of gold nanoparticles.

\section{Results and Discussion}

\subsection{Synthesis of Hyperbranched PEIs with Different Shells of Amide}

In the following modified HPEI10K with different amide shells such as acetic amide, propionic amide, butyric amide and isobutyric amide are labeled as HPEI10K-ACAm, HPEI10K-PRAm, HPEI10K-BUAm and HPEI10K-IBAm, respectively. Based on the commercially available HPEIs with $M_{\mathrm{n}}=10^{4} \mathrm{~g} / \mathrm{mol}$, namely HPEI10K, four hyperbranched polymers having different end groups were synthesized according to the similar procedure reported previously [26] and their structural parameters are summarized in Table 1. HPEI10K was modified with a covalently attached hydrophobic shell to create amphiphilic core-shell architectures with HPEI10K as a core and the amide acting as a shell (Scheme 1).

HPEI10K-XXAm $\mathrm{DF}_{\mathrm{DF}}$ can be achieved via addition of anhydride to a solution of PEI10K in chloroform. The degree of functionalization ( $\mathrm{DF}=$ number of shell molecules/number of the primary and secondary amines of HPEI, given in \%) was determined via integration of the corresponding ${ }^{1} \mathrm{H}$ NMR signals (Figure S1). In order to compare the difference of the HPEIs with different amide shells on the catalytic activity, the DF of every modified HPEI was almost the same (Table 1). 
Table 1. Structural parameters of modified hyperbranched polyethylenimine (HPEI)10K polymers.

\begin{tabular}{|c|c|c|c|c|}
\hline Polymer & $\begin{array}{l}M_{n} \text { of } \\
\text { HPEI Core }\end{array}$ & $\begin{array}{c}\text { Degree of } \\
\text { Functionalization a }(\%)\end{array}$ & $M_{\mathrm{n}(\mathrm{NMR})}{ }^{\mathrm{b}}\left(\times 10^{4}\right)$ & General Formula \\
\hline 1 & 10000 & 80 & 1.570 & HPEI10K-ACAm 0.80 \\
\hline 2 & 10000 & 82 & 1.785 & HPEI10K-PRAm $_{0.82}$ \\
\hline 3 & 10000 & 83 & 1.993 & HPEI10K-BUAm 0.83 \\
\hline 4 & 10000 & 80 & 1.950 & HPEI10K-IBAm 0.80 \\
\hline
\end{tabular}

a Degree of functionalization is the ratio of amide groups to the primary and secondary amines of HPEI, calculated from the ${ }^{1} \mathrm{H}$ NMR spectrum of the corresponding polymer; ${ }^{b}$ Molecular weights were calculated from the ${ }^{1} \mathrm{H}$ NMR spectra.

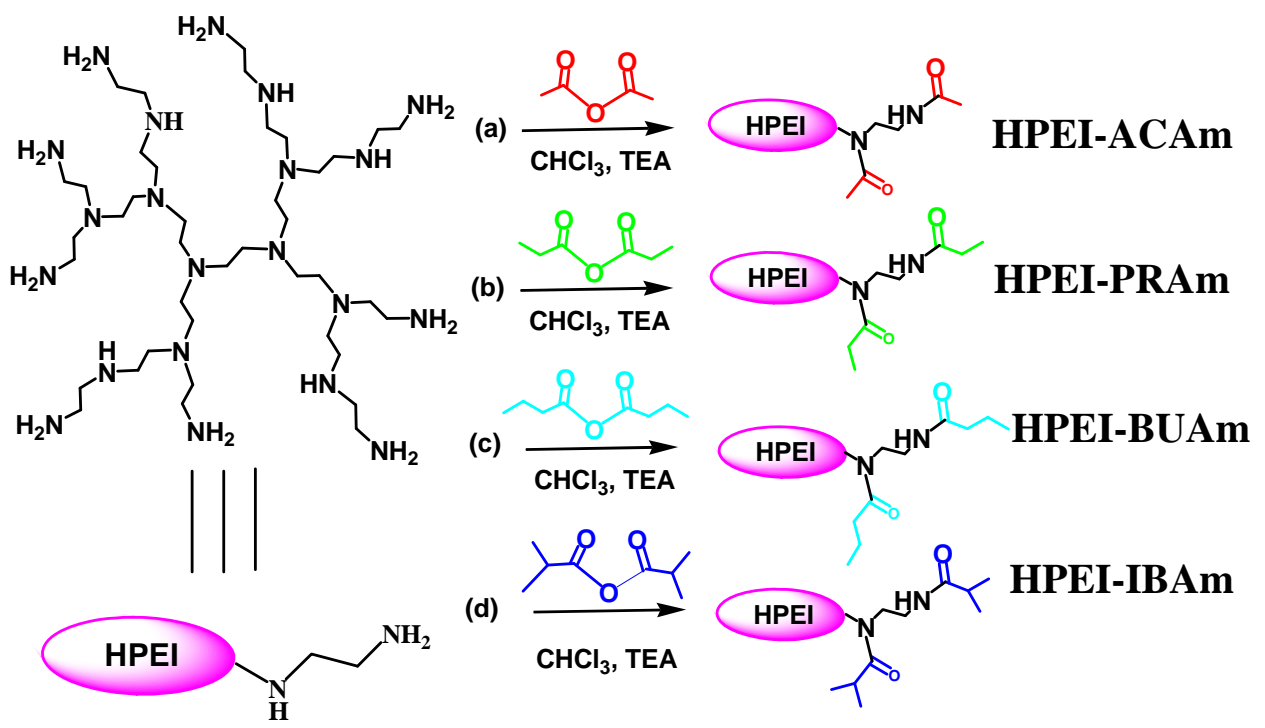

Scheme 1. Structure of modified hyperbranched polymers. Functionalization of HPEI10K with (a) acetic anhydride; (b) propionic anhydride; (c) butyric anhydride; and (d) isobutyric anhydride.

\subsection{Preparation of AuNPs and HPEI10K-XXAm Composites}

In order to prepare AuNPs with a different stabilizer, the obtained HPEI-XXAm polymers were mixed together with the anionic citrate-protected average 14-nm diameter AuNPs in water [27]. After being mixed with the HPEI-XXAm polymers, the AuNPs were all monodisperse and uniform characterized by TEM without staining (Figure 1). Previous experiments revealed that the gold nanoparticles can interact with the thermoresponsive HPEI-IBAm polymers [5]. It was reported that amino groups are weak ligands for gold, which adsorb onto the surface of AuNPs due to the non-covalent interaction of amino groups with the anionic citrates capping on AuNPs, but not to the displacement of citrates by amino groups [28]. Thus, it can be deduced that two interaction modes may contribute to the formation of the AuNPs composites. One is the ionic interaction between the partially protonated amino groups of HPEI-IBAm polymers and the negatively charged surface of AuNPs. The other is the hydrogen-bonding interaction between the negatively charged surface of AuNPs and the hydrogen atoms of the secondary amide and unreacted secondary amine groups of HPEI-IBAm polymers. In this sense, the AuNPs could composite with modified HPEIs which have the different amide shells. To provide evidence for the formation of the polymer layer on the surface of the Au NPs, we used EDX and NMR to determine the composition of the precipitates, which were obtained by five cycles of centrifugation of a solution of HPEI-IBAm-AuNPs (the free HPEI-IBAm was removed). Based on the EDX (Figure S2) and NMR (Figure S3) analysis, the C, N, and O content in the precipitates strongly supports the idea that the HPEI-IBAm polymers are indeed adsorbed onto the surface of the citrate-capped AuNPs. 


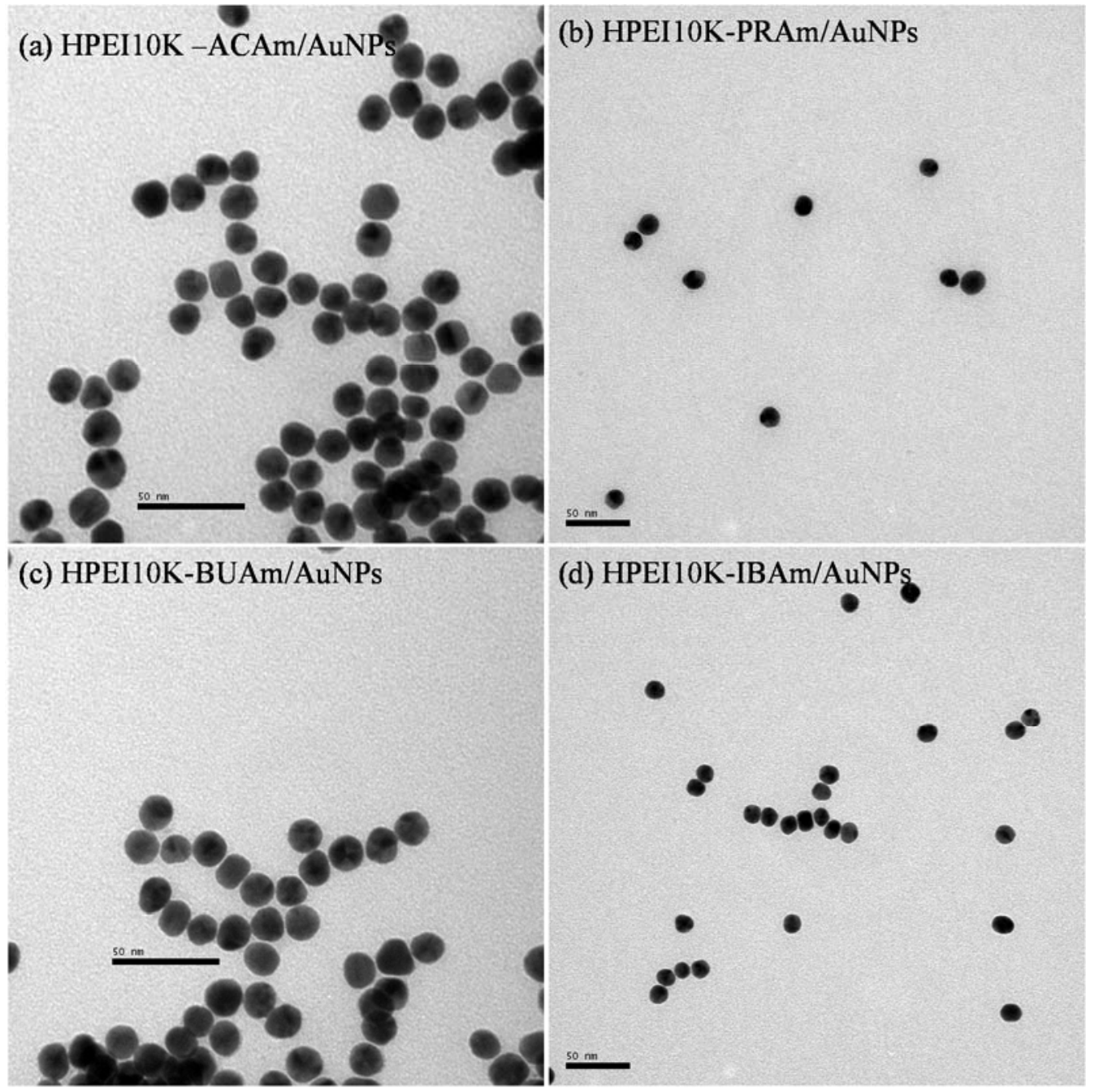

Figure 1. TEM images of composites of AuNPs with (a) HPEI10K-ACAm; (b) HPEI10K-PRAm; (c) HPEI10K-BUAm and (d) HPEI10K-IBAm.

\subsection{Comparison of the Catalytic Activity of AuNPs Capping with Different Protectors HPEI10K-XXAm}

After the discussion of the synthesis and the morphology of the gold nanopartices, the catalytic properties of these AuNPs composites were then investigated. The reduction of 4-nitrophenol with an excess of $\mathrm{NaBH}_{4}$ was used as a model reaction, of which the kinetics could be monitored by UV-VIS spectroscopy (Figure S4). After mixing 4-nitrophenol with $\mathrm{NaBH}_{4}$ in water, a yellow solution was obtained, and the UV-VIS spectrum showed a maximum absorbance peak centered at 400 $\mathrm{nm}$ due to the formation of 4-nitrophenolate ions [5]. The rates of reduction were assumed to be independent of the concentration of the $\mathrm{NaBH}_{4}$ since the reagent was used in large excess compared to 4-nitrophenol. Thus, the kinetic data was fitted with a first-order rate law. The ratio of absorbance $A_{\mathrm{t}}$ of 4-nitrophenolate at time $t$ to its value $A_{0}$ at $t=0$ (i.e. $\mathrm{A}_{\mathrm{t}} / \mathrm{A}_{0}$ ) can be directly interpreted as the ratio of the respective concentrations $C_{t} / C_{0}$. Therefore, the reaction conversion at time $t$ can be calculated according to Equation (1).

$$
\text { Conversion }(\%)=\left(1-C_{\mathrm{t}} / C_{0}\right) \times 100=\left(1-A_{\mathrm{t}} / A_{0}\right) \times 100
$$


At the same time, the reduction rate kinetics can be treated as a pseudo-first-order in the concentration of 4-nitrophenolate [29,30] according to Equation (2).

$$
\mathrm{d} C_{t} / \mathrm{d} t=-k_{\mathrm{app}} C_{t} \text { or } \ln \left(C_{\mathrm{t}} / C_{0}\right)=\ln \left(A_{\mathrm{t}} / A_{0}\right)=-k_{\mathrm{app}} t
$$

where $k_{\text {app }}$ is the apparent rate constant and the $C_{t}$ is the 4-nitrophenol concentration of at time $t$. This allows us to compare systems with different kinds of AuNPs composites. Firstly, the effect of HPEI-XXAm polymers on the AuNPs catalyzed reduction reaction was studied at $25{ }^{\circ} \mathrm{C}$. From Figure 2, it is clear that capping the AuNPs with HPEI-IBAm polymers having DF $=80 \%$ $($ HPEI10K-IBAm 0.80 ) catalyzed the reactions ( $\Delta$ curves in Figure 2$)$ about $32 \mathrm{~min}$. When capping

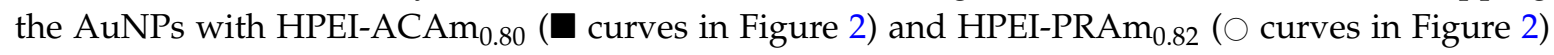
polymers, which have a similar DF, catalyzed the reactions at about $72 \mathrm{~min}$ and $136 \mathrm{~min}$, respectively. However, the conversion of the reaction in the presence of HEPI10K-BUAm 0.83 polymers remained unchanged after two days, indicating that it could not catalyze the reaction.
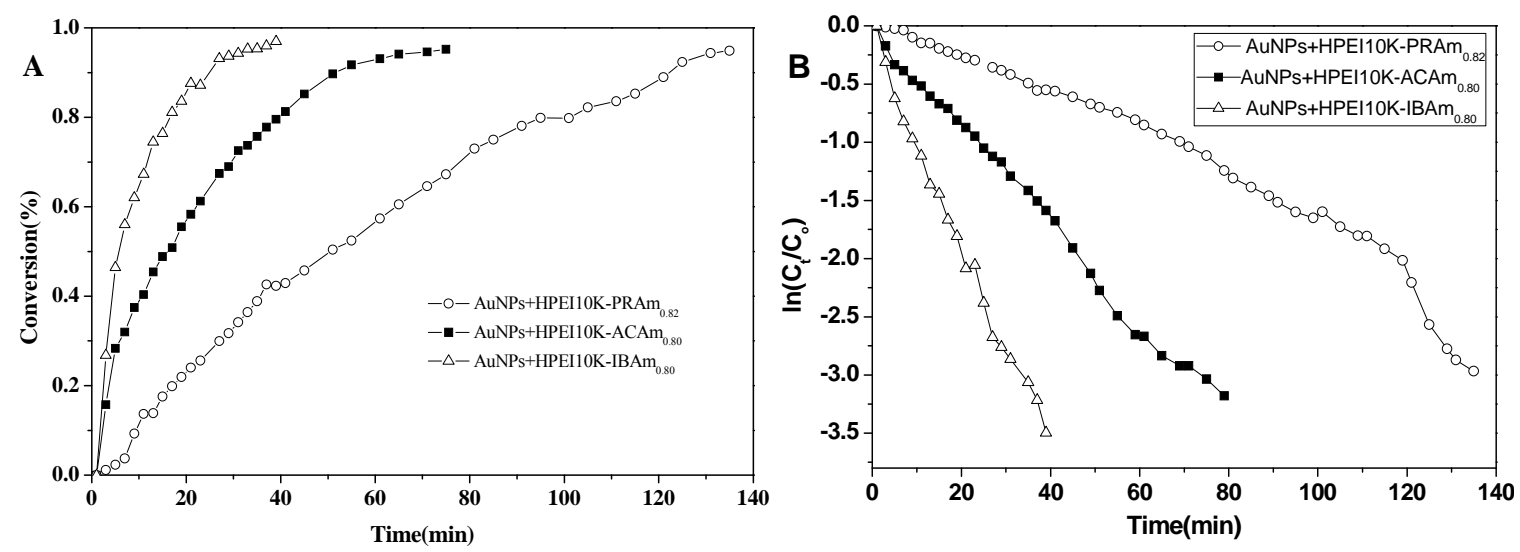

Figure 2. The AuNPs catalyzed reduction of 4-nitrophenol by $\mathrm{NaBH}_{4}$ in the presence of different polymers at $25{ }^{\circ} \mathrm{C}(\mathbf{A})$ plots of conversion vs. time; $(\mathbf{B})$ plots of $\ln \left(C_{t} / C_{0}\right)$ vs. time. ([4-nitrophenol] $=$ $1.0 \times 10^{-4} \mathrm{M},\left[\mathrm{NaBH}_{4}\right]=1.0 \times 10^{-2} \mathrm{M},[\mathrm{Au}]=1.42 \times 10^{-5} \mathrm{M},[$ HPEI-XXAm $\left.]=2.83 \times 10^{-6} \mathrm{M}\right)$.

From Figure 2A, it can be further interpreted that the rate was decreased with the increase of end alkyl chain length (not including the one ended branched chain structure). The $k_{\text {app }}$ values of HEPI10K-IBAm 0.80, HPEI10K-ACAm $_{0.80}$ and HPEI10K-PRAm 0.82 are $1.49 \times 10^{-3}, 0.69 \times 10^{-3}$ and $0.37 \times 10^{-3}$, respectively, according to Equation (2) and Figure 2B. It has been known that the reduction rate was usually controlled by the diffusion of 4-nitrophenolate to the surface of AuNPs [31,32]; thus, it was supposed that the presence of HPEI-XXAm polymers on the surface of AuNPs might change the diffusion speed of reactant 4-nitrophenolate ions due to the steric hindrance effect. The longer the carbon chain of the shell is, the stronger the steric hindrance is, causing 4-nitrophenolate ions to diffuse to the surface of AuNPs more slowly. The above shows that the catalytic activities depend on the length and structure of the carbon chain. It was found that the order of reactivity is HEPI10K-IBAm $0_{0.80}>$ HPEI10K-ACAm $0.80>$ HPEI10K-PRAm $_{0.82}>$ HPEI10K-BUAm 0.83 . It is easy to understand that the rate is HPEI-ACAm $0.80>$ HPEI-PRAm ${ }_{0.82}$ $>$ HPEI-BUAm 0.83 because of the reverse steric effect HPEI-ACAm $0.80<$ HPEI-PRAm $_{0.82}<$ HPEI-BUAm ${ }_{0.83}$. However, it is interesting that although HPEI modified with isobutyl has large steric hindrance, its catalytic rate is faster than that of ethyl. The surface of gold nanoparticles can only be capped with less HPEI-IBAm, owing to the isobutyl branched structure having large space volume. To prove the assumption, we analyzed the elemental composition of the precipitates obtained by five cycles of centrifugation of a solution of AuNPs-HPEI10K-IBAm 0.80 and AuNPs-HPEI10K-ACAm 0.80 using elemental analyses. Based on the nitrogen content of the composites in Table S1, the 
weights of nitrogen in AuNPs-HPEI10K-IBAm0.80 and AuNPs-HPEI10K-ACAm 0.80 are $16.24 \%$ and $23.31 \%$, respectively. This result indicates that the surface of AuNPs can be capped with less HPEI10K-IBAm 0.80 than HPEI10K-ACAm 0.80 . Therefore, it is beneficial for the diffusion of 4-nitrophenolate ions to the surface of AuNPs because of the lower polymer density on the periphery of gold nanoparticles. On the other hand, the space volume of the straight-chain alkyls is relatively small. Thus, the more modified polymers will act on the AuNPs surface, which will make the carbon chain arrangement be more compact and the reactant diffusion be difficult. The significant phenomenon of the reduction reaction rate is explained tentatively as follows: the steric hindrance effect of slowing the diffusion rate of 4-nitrophenolate by HPEI-XXAm polymers still existed in the system; however, large end group density also reduces the effective collision chance of AuNPs with 4-nitrophenolate. HPEI-IBAm polymers contain isobutyl branched structure having large space volume, thus the end group density is low, enhancing the collision chance of AuNPs with 4-nitrophenolate anions, resulting in the higher reaction rate.

\subsection{Effect of Temperature on the Catalytic Activity of the Reduction of 4-Nitrophenolate}

Figure 3 shows the dependence of the rate of reaction on temperature. Obviously, the change of temperature can change the catalytic activity of the gold nanparticles. From Figure 3, it can be seen that raising the reaction temperature can effectively accelerate the reaction at the beginning; however, when the reaction temperature arrives at close to $30{ }^{\circ} \mathrm{C}$, the reaction rate of the AuNPs-HPEI10K-IBAm 0.80 catalysts starts to decrease sharply with the further raising of the reaction temperature. From the classical Arrhenius equation, it can be learned that $k_{\text {app }}$ values usually increase with raising the reaction temperature, which has been observed in the reduction of 4-nitrophenol catalyzed by other AuNPs systems [30]. This abnormal phenomenon observed by us can be ascribed to the thermoresponsive property of the employed AuNP composites. Obviously, the change of temperature not only changes the catalytic activity of the gold nanoparticles but also leads to a shrinking of the HPEI10K-IBAm 0.80 network, which is in consistent with the results in our previous research [5]. Figure 3 also displays that the $k_{\text {app }}$ values of HPEI-ACAm 0.80 and HPEI-PRAm ${ }_{0.82}$ system increase with increasing the reaction temperature. As a consequence of this, the change of the rate constant is similar to the classical laws. Raising the reaction temperature, both HPEI-ACAm 0.80 and HPEI-PRAm 0.82 systems have higher catalytic performance. From the classical Arrhenius equation, it can be known that $k_{\text {app }}$ values usually increase with raising the reaction temperature, which has been observed in the reduction of 4-nitrophenol in other AuNP systems [5,30]. However, the conversion of the reaction in the presence of HEPI10K-BUAm 0.83 polymers remained unchanged until $35{ }^{\circ} \mathrm{C}$, meaning that it could not catalyze the reaction even at higher temperature. It can be explained that although high temperature usually can accelerate the reaction, the hydrophobic interaction is also strengthened due to the longer carbon chain of the shell. We found that the HEPI10K-BUAm ${ }_{0.83}$ solution had become turbid at $35{ }^{\circ} \mathrm{C}$, further indicating that the obtained AuNPs composites were thermoresponsive. Both the hydrophobic interaction and thermoresponsive property could decelerate the diffusion rate of the reactants onto the surface of AuNPs and counteract the acceleration by the temperature increase. 


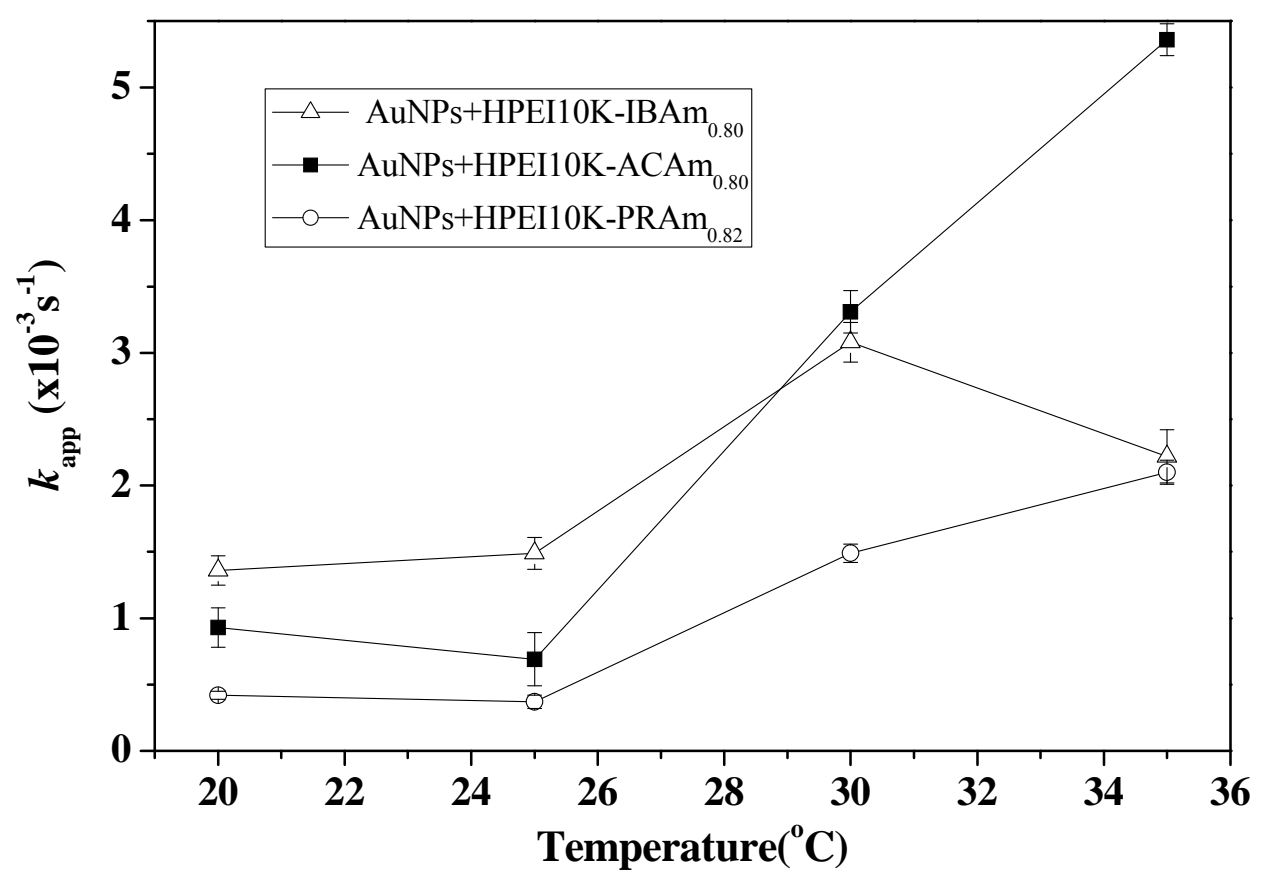

Figure 3. The plots of $k_{a p p}$ vs. time of the AuNPs catalyzed reduction of 4-nitrophenol by $\mathrm{NaBH}_{4}$ in the presence of different polymers at different temperature. ([4-nitrophenol] $=1.0 \times 10^{-4} \mathrm{M},\left[\mathrm{NaBH}_{4}\right]$ $=1.0 \times 10^{-2} \mathrm{M},[$ HPEI-IBAm $\left.]=2.83 \times 10^{-6} \mathrm{M}[\mathrm{Au}]=1.42 \times 10^{-5} \mathrm{M}\right)$.

\subsection{Effect of Gold Composites Concentration on the Catalytic Activity of the Reduction of 4-Nitrophenolate}

The catalytic activities of different composites concentration obtained from our study are summarized in Figure 4. Figure 4A shows the typical plots of conversion versus time with different concentrations of AuNPs-HPEI10K-IBAm 0.80 . It is clear that the higher composite concentration leads the reaction to reach more than $95 \%$ conversion in a shorter time. The reaction kinetic curves of $\ln \left(C_{t} / C_{0}\right)$ versus time are shown in Figure $4 \mathrm{~B}$. The reaction with high composite concentration is fast and the corresponding kinetic plots can be obtained according to Equation (2), and the results are shown in Figure 4C. From Figure 4C, it is clear that $k_{\text {app }}$ value decreases with lowering the composite concentration, and the $k_{\text {app }}$ of AuNPs-HPEI10K-IBAm 0.80 system is higher than HPEI10K-ACAm 0.80, HPEI10K-PRAm 0.82 and HPEI10K-BUAm 0.83 . The turnover frequency (TOF) values of the corresponding reactions were also calculated according to Equation (3):

$$
\mathrm{TOF}=[4-\text { nitrophenolate }] \times \text { conversion } /([\mathrm{Au}] \times t)
$$

Here, the concentration of 4-nitrophenolate was fixed to be $1.0 \times 10^{-4} \mathrm{M}$, and the conversion at time $t$ can be read from the plots of conversion vs. time. We estimated the TOF values for all the runs with the conversion of 4-nitrophenolate at 95\% and the results were plotted in Figure 4D. It is obvious that the TOF values of AuNPs-HPEI10K-IBAm 0.80 and AuNPs-HPEI10K-ACAm 0.80 system increase nearly linearly with raising the composite concentrations in the studied range and the maximum TOF is about $90 \mathrm{~h}^{-1}$ at $25{ }^{\circ} \mathrm{C}$, much superior to the $15.5 \mathrm{~h}^{-1}$ at $25{ }^{\circ} \mathrm{C}$ showed by another type of thermoresponsive AuNPs formed by the block copolymer poly(N-isopropylacrylamide)-b-poly(4-vinyl pyridine) self-assembled micelle with $2-4 \mathrm{~nm}$ diameter AuNPs [29]. The TOF values of our system are also comparable or even superior to the catalysts of gold dendrimer nanocomposites [31,32] and AuNPs immobilized with anion exchange resin [30] reported by Esumi et al. and Pal et al, respectively. 

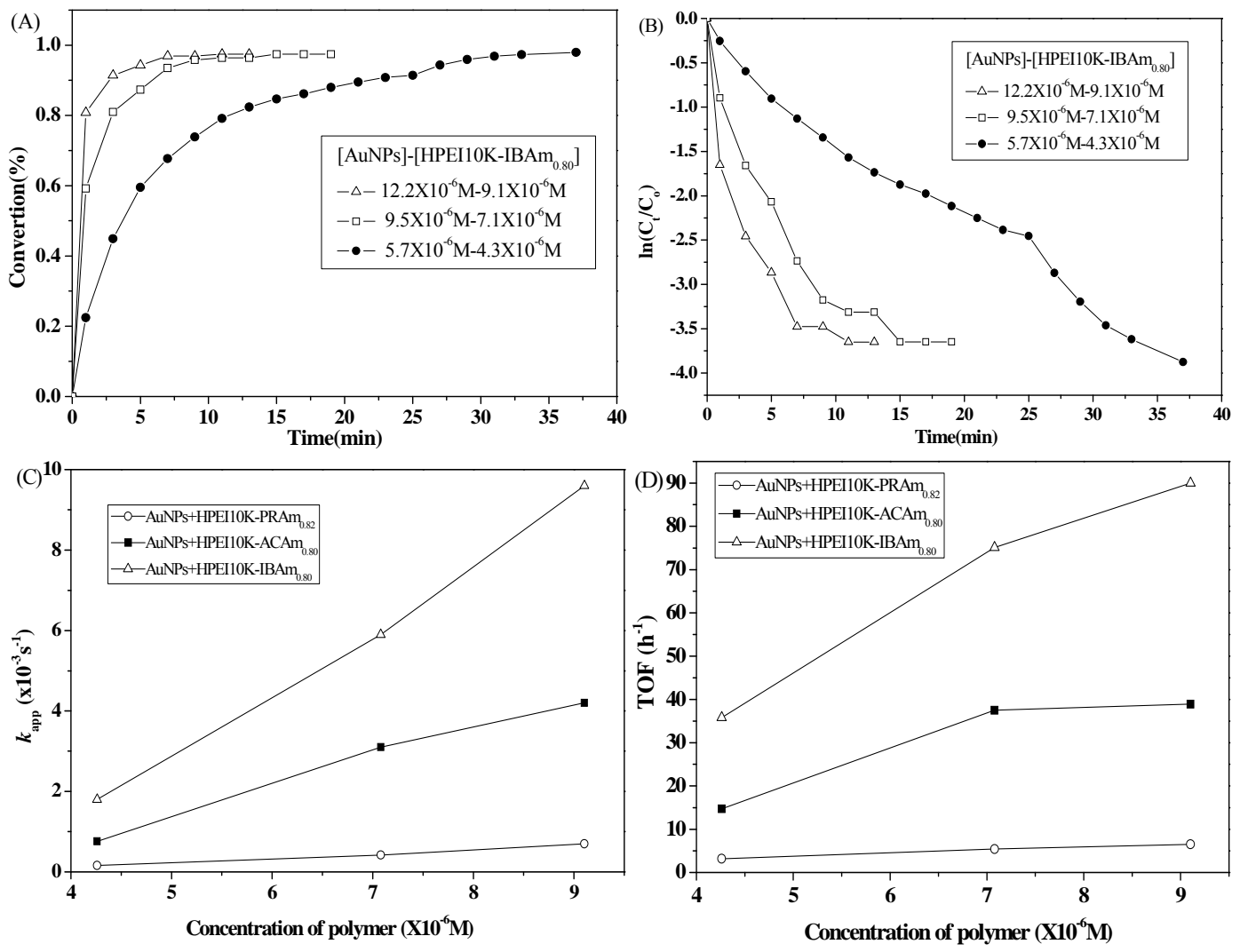

Figure 4. The reduction reaction of 4-nitrophenol by $\mathrm{NaBH}_{4}$ catalyzed by the composites of AuNPs with different amounts of HPEI-XXAm polymers at $25^{\circ} \mathrm{C}$. (A) plots of conversion vs. time; (B) plots of $\ln \left(C_{t} / C_{0}\right)$ vs. time; (C) plots of $k_{a p p} v$ s. concentration of polymer; (D) plots of TOF vs. concentration of polymer. ([4-nitrophenol] $=1.0 \times 10^{-4} \mathrm{M}$, $\left[\mathrm{NaBH}_{4}\right]=1.0 \times 10^{-2} \mathrm{M},[\mathrm{Au}]:[\mathrm{HPEI}-\mathrm{IBAm}]=1.34$ ).

\subsection{Effect of HPEI10K-XXAm Polymers Concentration on the Catalytic Activity of the Reduction} of 4-Nitrophenolate

Further study on the concentration effect of HPEI-XXAm polymers on the reduction rate was performed. Table 2 shows the $k_{\text {app }}$ and TOF with different concentrations of HPEI-XXAm polymers. It is clear that $k_{\text {app }}$ value and TOF can increase significantly with the rising of the polymer concentration.

Table 2. Results concerning the reduction of 4-nitrophenolate by gold nanoparticles (AuNPs)-HPEI-XXAm at $25^{\circ} \mathrm{C}$ (XXAm means ACAm, PRAm, BUAm or IBAm).

\begin{tabular}{|c|c|c|c|c|c|}
\hline \multirow{2}{*}{$\begin{array}{l}\text { Concentration of } \\
\text { AuNPs }\left(\times 10^{-6} \mathrm{M}\right)\end{array}$} & \multirow{2}{*}{$\begin{array}{c}\text { Concentration of } \\
\text { Polymer }\left(\times 10^{-6} \mathrm{M}\right)^{\text {a }}\end{array}$} & \multicolumn{2}{|c|}{$k\left(\times 10^{-3}\right)$} & \multicolumn{2}{|c|}{ TOF } \\
\hline & & $k_{\mathrm{AC}} \mathrm{b}$ & $k_{\mathrm{IB}} \mathrm{c}$ & TOF $_{A C}{ }^{d}$ & TOF $_{\text {IB }}{ }^{e}$ \\
\hline \multirow{2}{*}{5.69} & 4.26 & 0.760 & 1.80 & 14.7 & 35.8 \\
\hline & 8.50 & 1.98 & 2.80 & 38.5 & 55.6 \\
\hline \multirow{2}{*}{12.2} & 6.07 & 2.60 & 5.10 & 24.6 & 46.7 \\
\hline & 9.10 & 4.20 & 9.60 & 38.9 & 90.0 \\
\hline
\end{tabular}

${ }^{a}$ Concentration of corresponding polymer; ${ }^{\mathrm{b}} k_{\mathrm{AC}}$ : rate constant of AuNPs-HPEI10K-ACAm0.80 system; ${ }^{\mathrm{c}} k_{\mathrm{IB}}$ : rate constant of AuNPs-HPEI10K-IBAm0.80 system; ${ }^{\mathrm{d}} \mathrm{TOF}_{\mathrm{AC}}$ : the TOF value of AuNPs-HPEI10K-ACAm0.80 system; e $\mathrm{TOF}_{\mathrm{IB}}$ : the TOF value of AuNPs-HPEI10K-IBAm0.80 system. (all concentrations are fixed: [4-nitrophenolate] $=1.0 \times 10^{-4} \mathrm{M},\left[\mathrm{NaBH}_{4}\right]=1.0 \times 10^{-2} \mathrm{M}$ ).

The effect of HPEI10K-XXAm polymer concentration on the catalytic activity of the reduction of 4-nitrophenolate agrees with our previous work [5]. Briefly, higher amounts of HPEI-XXAm polymers can neutralize more negative charges of AuNPs surface, enhancing the collision chance 
of AuNPs with 4-nitrophenolate anions. The other factors such as the more electrostatic interaction and hydrogen-bonding interaction between HPEI-XXAm polymers and 4-nitrophenolate anions are also convenient for 4-nitrophenolate anions to diffuse to the surface of AuNPs. These factors might be dominant in controlling the reduction reaction rate, resulting in higher catalytic activity shown by system with higher polymer concentration.

\subsection{Effect of HPEI10K-XXAm Polymers Concentration on the Catalytic Activity of the Reduction of 4-Nitrophenolate by 8-nm Gold Nanoparticles}

Further study on the concentration effect of HPEI-XXAm polymers on the reduction rate by 8-nm gold nanoparticle was performed. Figure 5 shows the $k_{\text {app }}$ versus time with different concentrations of AuNPs-HPEI10K-XXAm. It has been known that catalytic activity is better with decrease in nanoparticle size. As expected, significant catalytic activity change was observed in AuNPs-HPEI10K-BUAm 0.83 system. Although reaction can be catalyzed by the AuNPs-HPEI10K-BUAm 0.83 system, the $k_{\text {app }}$ is relatively small. As shown in Figure 5, the order of reaction rate is still AuNPs-HEPI10K-IBAm $0.80>$ AuNPs-HPEI10K-ACAm $0.80>$ AuNPs-HPEI10K-PRAm $0.82>$ AuNPs-HPEI10K-BUAm $_{0.83}$, which is not dependent on the nanoparticle size but on the steric effect. In other words, the catalytic activity can be controlled by adjusting the different shells of the hyperbranched polyethylenimine.

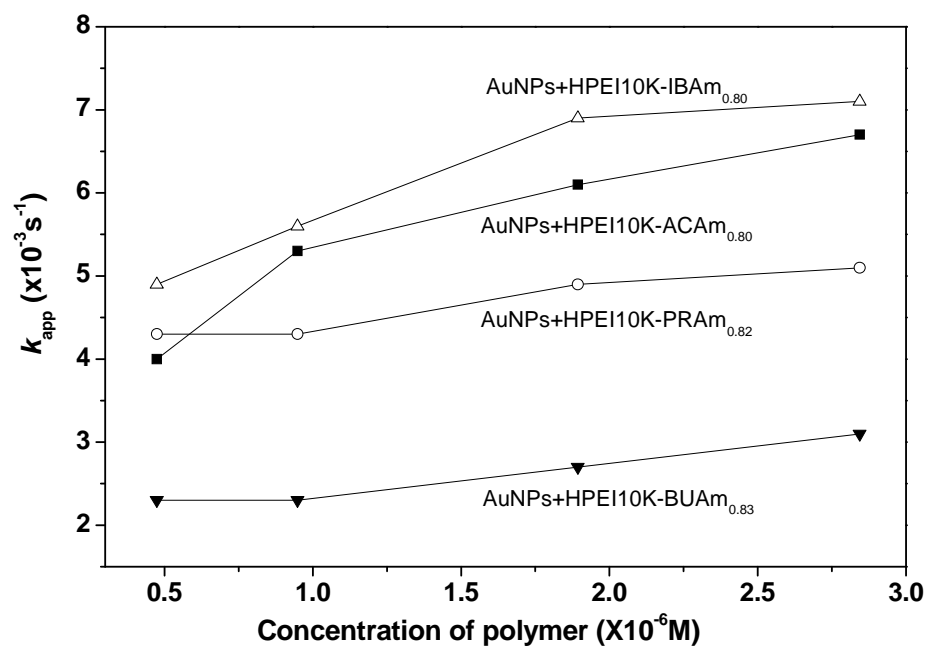

Figure 5. The plots of $k_{a p p}$ vs. time vs. time of the AuNPs catalyzed reduction of 4-nitrophenol by $\mathrm{NaBH}_{4}$ in the presence of different AuNPs-polymer concentration at $25{ }^{\circ} \mathrm{C}$. ([4-nitrophenolate $\left.]=1.0 \times 10^{-4} \mathrm{M},\left[\mathrm{NaBH}_{4}\right]=1.0 \times 10^{-2} \mathrm{M},[\mathrm{Au}]:[\mathrm{HPEI}-\mathrm{IBAm}]=5\right)$.

\subsection{Effect of Temperature on the Catalytic Activity of the Reduction of 4-Nitrophenolate by 8-nm Gold Nanoparticles}

The influence of reaction temperature on the reaction rate was also studied in smaller-size gold nanoparticle systems. From Figure 6, it can be seen that raising the reaction temperature can effectively accelerate the reaction rate for AuNPs-HPEI10K-ACAm 0.80 and AuNPs-HPEI10K-PRAm 0.82 systems. For AuNPs-HEPI10K-IBAm 0.80 , raising the reaction temperature can efficiently accelerate the reaction at first, however, when the reaction temperature arrives at $25^{\circ} \mathrm{C}$, the $k_{\text {app }}$ has not obviously changed with the further raising of the reaction temperature. About the AuNPs-HPEI10K-BUAm 0.83 system, when the reaction temperature increases, the reaction accelerates at first. When the reaction temperature arrives at $30^{\circ} \mathrm{C}$, the reaction rate begins to decrease sharply with the further raising the reaction temperature. Likewise, HPEI10K-BUAm 0.83 has thermoresponsive property that can decelerate the diffusion rate of the reactants onto the surface of AuNPs and counteract the acceleration by the temperature increase. 


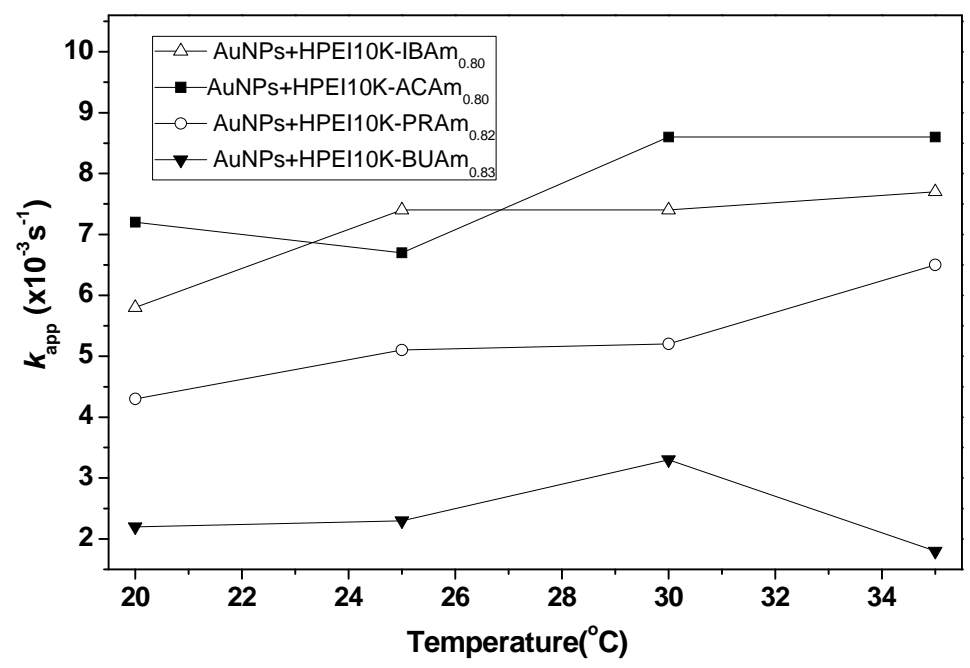

Figure 6. The plots of $k_{a p p}$ vs. time of the AuNPs catalyzed reduction of 4-nitrophenol by $\mathrm{NaBH}_{4}$ in the presence of different polymers at different temperature. ([4-nitrophenolate] $=1.0 \times 10^{-4} \mathrm{M}$, $\left[\mathrm{NaBH}_{4}\right]=1.0 \times 10^{-2} \mathrm{M}$, [HPEI-XXAm $\left.]=2.83 \times 10^{-6} \mathrm{M}[\mathrm{Au}]=1.42 \times 10^{-5} \mathrm{M}\right)$.

\section{Experimental Section}

\subsection{Chemicals}

Acetic anhydride, propionic anhydride, butyric anhydride, isobutyric anhydride $(98 \%$, Alfa Aesar, Haverhill, MA, USA) were used without further purification. Hyperbranched polyethylenimines, HPEI10K (Sigma-Aldrich, St. Louis, MO, USA, $M_{\mathrm{n}}=10000 \mathrm{~g} / \mathrm{mol}, M_{\mathrm{w}} / M_{\mathrm{n}}=2.5$ ) were dried under vacuum prior to use. Benzoylated cellulose tubing (Molecular Weight Cut Off 1000, Sigma-Aldrich, St. Louis, MO, USA), $\mathrm{HAuCl}_{4}$ (Tianjin Yinda Chemical company, Tianjin, China) and Sodium citrate (Tianjin Chemical Reagent Plant, Tianjin, China) wereused directly. De-ionized water was double-distilled before use. Triethylamine (TEA), sodium hydroxide, 4-nitrophenol, $\mathrm{NaBH}_{4}$ and potassium carbonate (Tianjin University Kewei Chemical Company, Tianjin, China) were used as received.

\subsection{Nomenclature}

XXAm represents ACAm, PRAm, BUAm or IBAm. ACAm, PRAm, BUAm and IBAm represent acetamide, propionamide, butyramide and isobutyramide, respectively. HPEI-XXAm HPEI terminated with plenty of amide groups, DF means the degree of amidation relative to the total reactive primary and secondary amines of HPEI.

\subsection{Synthesis of HPEIs with Different Shells}

The preparation of HPEIs with different shells is similar to the previous report [26]. It is exemplified for HPEI10K-ACAm 0.80 : Under nitrogen atmosphere, acetic anhydride $(2.11 \mathrm{~g}, 20.7 \mathrm{mmol})$ was added dropwise to the mixture of HPEI10K ( $1.52 \mathrm{~g}, 25.8 \mathrm{mmol}$ of terminal groups) and triethyl amine $(2.30 \mathrm{~g}$, $22.7 \mathrm{mmol}$ ) in $20 \mathrm{~mL}$ of chloroform at $0{ }^{\circ} \mathrm{C}$ with vigorous stirring. Subsequently, the reaction mixture was kept and carried out at room temperature for $24 \mathrm{~h}$. Then the reaction temperature was raised to $65^{\circ} \mathrm{C}$ for $2 \mathrm{~h}$. After cooling down to room temperature, the produced solide was filtered off. Volatiles in the filtrate were removed under vacuum and the residue was dissolved in $40 \mathrm{~mL}$ of methanol. About $1 \mathrm{~g}$ of potassium carbonate was added to the solution and the mixture was stirred at room temperature for $4 \mathrm{~h}$. After filtration, the solution was concentrated to $c a .10 \mathrm{~mL}$ and then purified by dialysis against methanol using a benzoylated cellulose membrane (MWCO $1000 \mathrm{~g} / \mathrm{mol}$ ) for two days. Finally, the methanol solvent was removed, and the product was dried in vacuum for $24 \mathrm{~h}$. 
The preparation of HPEIs with other anhydride is similar to the procedure of HPEI10K-ACAm 0.80 , only with different amount of substance. The corresponding quantity was as follows: propionic anhydride ( $2.85 \mathrm{~g}, 21.9 \mathrm{mmol})$, HPEI10K (1.61 g, $27.3 \mathrm{mmol}$ of terminal groups) and triethyl amine ( $2.44 \mathrm{~g}, 24.1 \mathrm{mmol})$; butyric anhydride ( $3.43 \mathrm{~g}, 21.7 \mathrm{mmol})$, HPEI10K (1.55 g, $26.4 \mathrm{mmol}$ of terminal groups) and triethyl amine (2.42 g, $23.9 \mathrm{mmol})$; isobutyric anhydride $(2.85 \mathrm{~g}, 18.0 \mathrm{mmol})$, HPEI10K $(1.37 \mathrm{~g}, 23.2 \mathrm{mmol}$ of terminal groups) and triethyl amine ( $2.07 \mathrm{~g}, 20.5 \mathrm{mmol})$.

\subsection{Preparation of Citrate Protected AuNPs}

Gold nanoparticles (AuNPs) were synthesized using the classical citrate reduction method [31]. The aqueous solution of $\mathrm{HAuCl}_{4}\left(4.27 \times 10^{-4} \mathrm{M}\right)$ was boiled gently under reflux. Sodium citrate $\left(1.34 \times 10^{-3} \mathrm{M}\right)$ was added under vigorous stirring to this solution. Within $4 \mathrm{~min}$, the solution turned out wine-red, indicating the formation of AuNPs. After $10 \mathrm{~min}$, the reaction vessel was removed from the heating element and allowed to cool to room temperature.

\subsection{Preparation of AuNPs Coated with Polymers}

HPEI-ACAm (1.4 mg), HPEI-PRAm (1.5 mg), HPEI-BUAm (1.6 mg) or HPEI-IBAm (1.6 mg) were dissolved in $5 \mathrm{~mL}$ of de-ionized water respectively. After adjusting the $\mathrm{pH}$ of the solution to the aimed one, $1 \mathrm{~mL}$ of the AuNPs solution was added dropwise to the polymer solution. The mixture was kept at room temperature for at least a half day before measurement.

\subsection{Preparation of Small Particle Size AuNPs Coated with Polymers}

HPEI-ACAm (9.08 mg), HPEI-PRAm (9.48 mg), HPEI-BUAm (10.58 mg) or HPEI-IBAm (10 mg) was dissolved in $2 \mathrm{~mL}$ of de-ionized water respectively. Then, the aqueous solution of $\mathrm{HAuCl}_{4}$ $\left(30.4 \mathrm{~mL}, 8.75 \times 10^{-5} \mathrm{M}\right)$ and $\mathrm{NaBH}_{4}\left(5 \mathrm{~mL}, 5.32 \times 10^{-3} \mathrm{M}\right)$ were added into the polymer solution. Immediately, the solution turned out yellowish-brown, indicating the formation of the small particle size AuNPs.

\subsection{Catalytic Reduction of 4-Nitrophenol by $\mathrm{NaBH}_{4}$}

The catalytic reduction was conducted in a standard quartz cuvette with a 1-cm path length. The aqueous solution of $\mathrm{NaBH}_{4}$ with a concentration of $0.30 \mathrm{M}(0.1 \mathrm{~mL})$ was mixed together with $2.7 \mathrm{~mL}$ of 4-nitrophenol aqueous solution $\left(1.1 \times 10^{-4} \mathrm{M}\right)$ and then the mixture was fixed to a preset temperature. Then $0.2 \mathrm{~mL}$ of the aqueous solution of the modified hyperbranched polyethylenimines-AuNPs composites with the same temperature was added immediately. The initial concentrations of 4-nitrophenol and $\mathrm{NaBH}_{4}$ were kept to be $1.0 \times 10^{-4} \mathrm{M}$ and $0.01 \mathrm{M}$, respectively, while the concentrations of $\mathrm{Au}$ and polymers were varied. The absorption spectra were recorded every $2 \mathrm{~min}$ in the range of 250 to $600 \mathrm{~nm}$.

\subsection{Characterization of AuNPs and Its Composites}

The AuNPs and AuNPs-composites were characterized by dynamic light scattering, UV-VIS spectroscopy and TEM. Dynamic light scattering was made on Zetasizer Nano-ZS90 (Malvern, London, UK). UV-VIS spectra were obtained from T6 UV-VIS Spectrophotometer (Beijing Purkinje General Instrument Co., Ltd. Beijing, China). TEM observation was made on a TECNAI G2 F20 (Philips, Amsterdam Holland) operated at $200 \mathrm{kV} .{ }^{1} \mathrm{H}$ NMR spectra were recorded on a INOVA $500 \mathrm{MHz}$ spectrometer (Varian, California, USA), operated at $500 \mathrm{MHz}$. The chemical shifts are given in parts per million (ppm).

\section{Conclusions}

In summary, we have reported on a simple protocol to prepare hyperbranched core-shell architectures with different shells of amide. Functionalized HPEI-XXAm molecules have been used as 
protectors for gold nanoparticles. Novel AuNP composites were prepared through the non-covalent interaction between HPEI-XXAm polymers and AuNPs. The catalytic activity of the AuNPs composites was monitored in different systems by the reduction of 4-nitrophenolate with $\mathrm{NaBH}_{4}$. The analysis of the kinetic data obtained herein together with data finished in the literature shows the following features: (i) the catalytic activity of the AuNPs mainly depends on the type of protector system used; (ii) the difference in catalytic activity of the AuNPs composites is due to the limitation of diffusion and the degree of carbon chain arrangement denseness; (iii) the order of reactivity is HEPI10K-IBAm $0.80>$ HPEI10K-ACAm $0.80>$ HPEI10K-PRAm $_{0.82}>$ HPEI10K-BUAm $_{0.83}$; (iv) raising the AuNPs-HPEI-XXAm concentration can increase both the reaction rate and TOF values for HEPI10K-IBAm ${ }_{0.80}>$ HPEI10K-ACAm 0.80 systems, but the TOF values of AuNPs-HPEI10K-PRAm 0.82 system do not change obviously; raising the concentrations of the capping HPEI-XXAm polymers can also increase both the reaction rate and TOF values; (v) increasing the reaction temperature accelerates the reaction rate for AuNPs-HPEI10K-ACAm 0.80 and AuNPs-HPEI10K-PRAm 0.82 systems; increasing the reaction temperature accelerates the reaction rate at the beginning but reached a plateau or decreased sharply for AuNPs-HPEI10K-IBAm 0.80 and AuNPs-HPEI10K-BUAm 0.82 systems because their corresponding AuNPs composites were thermoresponsive. As such, the catalysis experiments demonstrate the catalytic activity can be controlled by adjusting the different shell structures. Moreover, this work also exhibits potential for how carrier systems for AuNPs could be designed to adjust the catalytic activity.

Acknowledgments: This work was financially supported by the National Science Foundation of China (21304043, 51403097), the Natural Science Foundation of Shandong Province (ZR2012BQ024, 2014ZRB019WZ) National Training Programs of Innovation and Entrepreneurship for Undergraduates (201510451039, 201510451022) and Natural Science Foundation of Ludong University (LY2012003, LY2013010).

Author Contributions: All the authors contributed to the paper. Xunyong Liu and Yi Liu were involved in writing and designing the aim of this manuscript. Yi Liu, Li Xu and Mengnan Cao did the experiment.

Conflicts of Interest: The authors declare no conflict of interest.

\section{References}

1. Munnik, P.; de Jongh, P.E.; de Jong, K.P. Recent developments in the synthesis of supported catalysts. Chem. Rev. 2015, 115, 6687-6718. [CrossRef] [PubMed]

2. Zhang, Y.; Cui, X.; Shi, F.; Deng, Y. Nano-gold catalysis in fine chemical synthesis. Chem. Rev. 2012, 112, 2467-2505. [CrossRef] [PubMed]

3. Hashmi, A.S.K. Gold-catalyzed organic reactions. Chem. Rev. 2007, 107, 3180-3211. [CrossRef] [PubMed]

4. Daniel, M.-C.; Astruc, D. Gold nanoparticles: Assembly, supramolecular chemistry, quantum-size-related properties, and applications toward biology, catalysis, and nanotechnology. Chem. Rev. 2004, 104, 293-346. [CrossRef] [PubMed]

5. Liu, X.-Y.; Cheng, F.; Liu, Y.; Liu, H.-J.; Chen, Y. Preparation and characterization of novel thermoresponsive gold nanoparticles and their responsive catalysis properties. J. Mater. Chem. 2010, 20, 360-368. [CrossRef]

6. Astruc, D.; Boisselier, E.; Ornelas, C. Dendrimers designed for functions: From physical, photophysical, and supramolecular properties to applications in sensing, catalysis, molecular electronics, photonics, and nanomedicine. Chem. Rev. 2010, 110, 1857-1959. [CrossRef] [PubMed]

7. Zhao, M.; Crooks, R.M. Homogeneous hydrogenation catalysis with monodisperse, dendrimer-encapsulated pd and pt nanoparticles. Angew. Chem. Int. Ed. 1999, 38, 364-366. [CrossRef]

8. Esumi, K.; Isono, R.; Yoshimura, T. Preparation of PAMAM- and PPI-metal (silver, platinum, and palladium) nanocomposites and their catalytic activities for reduction of 4-nitrophenol. Langmuir 2004, 20, 237-243. [CrossRef] [PubMed]

9. Zhang, J.; Xu, S.; Kumacheva, E. Polymer microgels: Reactors for semiconductor, metal, and magnetic nanoparticles. J. Am. Chem. Soc. 2004, 126, 7908-7914. [CrossRef] [PubMed]

10. Bönnemann, H.; Brijoux, W.; Tilling, A.S.; Siepen, K. Application of heterogeneous colloid catalysts for the preparation of fine chemicals. Top. Catal. 1997, 4, 217-227. [CrossRef] 
11. Zhao, M.; Sun, L.; Crooks, R.M. Preparation of cu nanoclusters within dendrimer templates. J. Am. Chem. Soc. 1998, 120, 4877-4878. [CrossRef]

12. Mecking, S.; Thomann, R.; Frey, H.; Sunder, A. Preparation of catalytically active palladium nanoclusters in compartments of amphiphilic hyperbranched polyglycerols. Macromolecules 2000, 33, 3958-3960. [CrossRef]

13. Aymonier, C.; Schlotterbeck, U.; Antonietti, L.; Zacharias, P.; Thomann, R.; Tiller, J.C.; Mecking, S. Hybrids of silver nanoparticles with amphiphilic hyperbranched macromolecules exhibiting antimicrobial properties. Chem. Commun. 2002, 3018, 3018-3019. [CrossRef]

14. Mornet, S.; Vasseur, S.; Grasset, F.; Duguet, E. Magnetic nanoparticle design for medical diagnosis and therapy. J. Mater. Chem. 2004, 14, 2161-2175. [CrossRef]

15. Frederix, F.; Friedt, J.-M.; Choi, K.-H.; Laureyn, W.; Campitelli, A.; Mondelaers, D.; Maes, G.; Borghs, G. Biosensing based on light absorption of nanoscaled gold and silver particles. Anal. Chem. 2003, 75, 6894-6900. [CrossRef] [PubMed]

16. Liu, X.-Y.; Cheng, F.; Liu, Y.; Li, W.-G.; Chen, Y.; Pan, H.; Liu, H.-J. Thermoresponsive gold nanoparticles with adjustable lower critical solution temperature as colorimetric sensors for temperature, ph and salt concentration. J. Mater. Chem. 2010, 20, 278-284. [CrossRef]

17. Liu, Y.; Xu, L.; Liu, J.-S.; Liu, X. Simultaneous enrichment, separation and detection of mercury (II) ions using cloud point extraction and colorimetric sensor based on thermoresponsive hyperbranched polymer-gold nanocomposite. Anal. Methods 2015. [CrossRef]

18. Liu, Y.; Liu, Y.; Li, Z.; Liu, J.; Xu, L.; Liu, X. An unusual red-to-brown colorimetric sensing method for ultrasensitive silver(I) ion detection based on a non-aggregation of hyperbranched polyethylenimine derivative stabilized gold nanoparticles. Analyst 2015, 140, 5335-5343. [CrossRef] [PubMed]

19. Praharaj, S.; Nath, S.; Ghosh, S.K.; Kundu, S.; Pal, T. Immobilization and recovery of au nanoparticles from anion exchange resin: Resin-bound nanoparticle matrix as a catalyst for the reduction of 4-nitrophenol. Langmuir 2004, 20, 9889-9892. [CrossRef] [PubMed]

20. Campbell, C.T.; Parker, S.C.; Starr, D.E. The effect of size-dependent nanoparticle energetics on catalyst sintering. Science 2002, 298, 811-814. [CrossRef] [PubMed]

21. Moisan, S.; Martinez, V.; Weisbecker, P.; Cansell, F.; Mecking, S.; Aymonier, C. General approach for the synthesis of organic-inorganic hybrid nanoparticles mediated by supercritical $\mathrm{CO}_{2}$. J. Am. Chem. Soc. 2007, 129, 10602-10606. [CrossRef] [PubMed]

22. Poupart, R.; Le Droumaguet, B.; Guerrouache, M.; Carbonnier, B. Copper nanoparticles supported on permeable monolith with carboxylic acid surface functionality: Stability and catalytic properties under reductive conditions. Mater. Chem. Phys. 2015, 163, 446-452. [CrossRef]

23. Bronstein, L.M.; Shifrina, Z.B. Dendrimers as encapsulating, stabilizing, or directing agents for inorganic nanoparticles. Chem. Rev. 2011, 111, 5301-5344. [CrossRef] [PubMed]

24. Cho, T.J.; Zangmeister, R.A.; MacCuspie, R.I.; Patri, A.K.; Hackley, V.A. Newkome-type dendron-stabilized gold nanoparticles: Synthesis, reactivity, and stability. Chem. Mater. 2011, 23, 2665-2676. [CrossRef] [PubMed]

25. Liu, Y.; Fan, Y.; Yuan, Y.; Chen, Y.; Cheng, F.; Jiang, S.-C. Amphiphilic hyperbranched copolymers bearing a hyperbranched core and a dendritic shell as novel stabilizers rendering gold nanoparticles with an unprecedentedly long lifetime in the catalytic reduction of 4-nitrophenol. J. Mater. Chem. 2012, 22, 21173-21182. [CrossRef]

26. Liu, X.; Cheng, F.; Liu, H.; Chen, Y. Unusual salt effect on the lower critical solution temperature of hyperbranched thermoresponsive polymers. Soft Matter 2008, 4, 1991-1994. [CrossRef]

27. Kimling, J.; Maier, M.; Okenve, B.; Kotaidis, V.; Ballot, H.; Plech, A. Turkevich method for gold nanoparticle synthesis revisited. J. Phys. Chem. B 2006, 110, 15700-15707. [CrossRef] [PubMed]

28. Mayya, K.S.; Caruso, F. Phase transfer of surface-modified gold nanoparticles by hydrophobization with alkylamines. Langmuir 2003, 19, 6987-6993. [CrossRef]

29. Wang, Y.; Wei, G.; Zhang, W.; Jiang, X.; Zheng, P.; Shi, L.; Dong, A. Responsive catalysis of thermoresponsive micelle-supported gold nanoparticles. J. Mol. Catal. A 2007, 266, 233-238. [CrossRef]

30. Panigrahi, S.; Basu, S.; Praharaj, S.; Pande, S.; Jana, S.; Pal, A.; Ghosh, S.K.; Pal, T. Synthesis and size-selective catalysis by supported gold nanoparticles: Study on heterogeneous and homogeneous catalytic process. J. Phys. Chem. C 2007, 111, 4596-4605. [CrossRef] 
31. Esumi, K.; Miyamoto, K.; Yoshimura, T. Comparison of PAMAM-Au and PPI-Au nanocomposites and their catalytic activity for reduction of 4-nitrophenol. J. Colloid Interface Sci. 2002, 254, 402-405. [CrossRef] [PubMed]

32. Hayakawa, K.; Yoshimura, T.; Esumi, K. Preparation of gold-dendrimer nanocomposites by laser irradiation and their catalytic reduction of 4-nitrophenol. Langmuir 2003, 19, 5517-5521. [CrossRef]

(c) 2015 by the authors; licensee MDPI, Basel, Switzerland. This article is an open access article distributed under the terms and conditions of the Creative Commons by Attribution (CC-BY) license (http:/ / creativecommons.org/licenses/by/4.0/). 